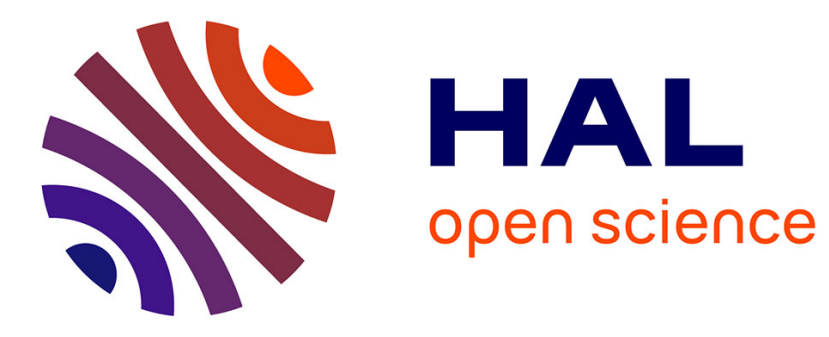

\title{
Design of spatial microphone arrays for sound field interpolation
}

Gilles Chardon, Wolfgang Kreuzer, Markus Noisternig

\section{To cite this version:}

Gilles Chardon, Wolfgang Kreuzer, Markus Noisternig. Design of spatial microphone arrays for sound field interpolation. IEEE Journal of Selected Topics in Signal Processing, 2015, pp.11. 10.1109/JSTSP.2015.2412097 . hal-01148971

\section{HAL Id: hal-01148971 \\ https://hal.inria.fr/hal-01148971}

Submitted on 6 May 2015

HAL is a multi-disciplinary open access archive for the deposit and dissemination of scientific research documents, whether they are published or not. The documents may come from teaching and research institutions in France or abroad, or from public or private research centers.
L'archive ouverte pluridisciplinaire HAL, est destinée au dépôt et à la diffusion de documents scientifiques de niveau recherche, publiés ou non, émanant des établissements d'enseignement et de recherche français ou étrangers, des laboratoires publics ou privés. 


\title{
Design of spatial microphone arrays for sound field interpolation
}

\author{
Gilles Chardon, Member, IEEE, Wolfgang Kreuzer, and Markus Noisternig, Member, IEEE
}

\begin{abstract}
This article presents a design method for microphone arrays with arbitrary geometries. Based on a theoretical analysis and on the magic points method, it allows for the interpolation of a sound field in a generic convex domain with a limited number of microphones on a given frequency band. It is shown that only a few microphones are needed in the interior of the considered domain to ensure a low interpolation error in the frequency band of interest, and that most of the microphones have to be located on the boundary of the domain, with a nonuniform density depending on the shape of the domain. Practical design constraints can be included in the optimization process. Comparisons for some particular array geometries with design methods known from the literature are given, showing that the proposed approach results in lower errors.
\end{abstract}

Index Terms-sound field analysis, array processing, microphone arrays, numerical robustness

\section{INTRODUCTION}

$\mathbf{T}$ HIS paper deals with the spatial interpolation of sound fields by microphone arrays of general shapes. Although the interpolation error is seldom-used as a performance measure for microphone arrays, its use can be justified by the following two main arguments. Firstly, theoretical results on interpolation and sampling of functions are widely available, and are the subject of ongoing research (see e.g. compressed sensing). Secondly, a microphone array that is able to interpolate the sound field in some spatial domain should be able to simulate the measurements of any other microphone array in the same domain. It thus should perform similarly in terms of different array performance measures, such as the white noise gain, the condition number, and the estimation of the spherical harmonics expansion coefficients.

Spherical microphone arrays are upon the most widely used array geometries for 3-D sound field recording. Early studies on open spherical microphone arrays have shown that numerical instabilities appear at some wave numbers, which

Copyright (c) 2014 IEEE. Personal use of this material is permitted. However, permission to use this material for any other purposes must be obtained from the IEEE by sending a request to pubs-permissions@ieee.org

G. Chardon was with the Acoustics Research Institute, Austrian Academy of Sciences, Vienna, during part of the preparation of this article, and is now with the Laboratoire des Signaux et Systèmes (L2S), CentraleSupélec, CNRS UMR 8506, Univ. Paris Sud, Gif-sur-Yvette, France (e-mail: gilles.chardon@centralesupelec.fr).

W. Kreuzer is with the Acoustics Research Institute, Austrian Academy of Sciences, Vienna, Austria (e-mail: wolfgang.kreuzer@oeaw.ac.at)

M. Noisternig is with the Acoustic and Cognitive Spaces Research Group at IRCAM, CNRS, Sorbonne Universités, UPMC Université Paris 6, UMR 9912 STMS, Paris, France (e-mail: markus.noisternig@ircam.fr).

Parts of the preliminary work related to the current manuscript appeared in the Proceedings of the IEEE International Conference on Acoustics, Speech, and Signal Processing (ICASSP 2014). are related to the roots of the spherical Bessel functions (cf. [1], [2]). Meyer and Elko [3] proposed to overcome this problem by placing the microphones on a rigid, sound hard sphere. This approach is not very well suited for arrays with large radii and several authors have proposed alternative array geometries. One way to increase the stability around the roots of the Bessel functions, without the drawback of introducing a rigid sphere into the measured sound field, is to use cardioid microphones facing outwards in radial direction (cf. [4], [5], [6]). In practice, this approach is difficult to realize, since cardioid microphones have a relatively high noise level at low frequencies. This solution is also prone to microphone positioning and steering errors, as well as to imperfections in the microphone directivity patterns. Double-sphere arrays provide an alternative solution to the ill-conditioning problem of open-sphere arrays (cf. [6], [7], [8]). They typically consist of pressure microphones arranged on two concentric spheres (i.e. two open spheres or, alternatively, an inner rigid sphere plus an outer open sphere) with different radii. This approach is particularly well suited for arrays with a large aperture that also covers the lower frequency range. The main drawback of a double-sphere array is that it requires at least twice the number of microphones of a single-sphere array.

Some authors proposed the use of non-spherical array geometries for increasing the stability around the roots of the Bessel functions. Rafaely [9] showed that an open spherical shell array (i.e. where some microphones are moved from the boundary to the inside of the sphere) can achieve robustness on a large frequency band. The positions of the interior sampling points are determined by a constrained nonlinear optimization procedure that minimizes the maximal condition number of the matrix that contains the product of the spherical Bessel functions with the spherical harmonics. With this method it is, however, not straightforward to determine the number of interior sampling points that, for a given array order, ensure stability and convergence of the optimization routine. Abhayapala and Gupta [10] proposed a hybrid array geometry that uses pairs of circular arrays to sample the three-dimensional sound field. This approach puts lesser restrictions on sensor locations and allows for an increased operating bandwidth. Another attempt to overcome the problem of zero-valued Bessel functions is the double-sided cone array [11]. It exploits the radial orthogonality of the spherical Bessel functions, evaluated on the surface of a double-sided cone, to estimate the spherical harmonics expansion coefficients over a relatively wide frequency range. Anyway, it has been shown that the estimation fails for some frequencies. This can only be avoided by sampling the sound field at two or more cones and therefore 
requires a relatively large number of microphones. The spindle torus array [12] is obtained by projecting a uniform sample distribution on the sphere to a self-intersecting torus. This array achieves robustness against noise and can be easily implemented by a scanning microphone setup. However, the increased robustness comes for the expense of a relatively high number of required sampling nodes.

Mignot et al. [13] introduced an interpolation method for room impulse responses at low frequencies. This method approximates the sound field by sums of plane waves using measurements of the sound field on an array of randomly placed microphones. The design of the sampling grid was, however, not analyzed and is very likely suboptimal. In [14], it has been shown that for time-limited signals (such as room impulse responses) nonuniform sampling in the frequency domain can be used to avoid samples near the Bessel nulls. The optimal positions of the samples in the frequency domain can be numerically obtained by minimizing the condition number of the Fourier matrix and the diagonal matrix holding values of the spherical Bessel function.

In [15], Chardon et al. presented an optimal design (with respect to the number of sampling points) for open spherical microphone arrays by adding few microphones inside the sphere. The number and the positioning of these microphones are dependent on the eigenmodes of the sphere in the wavenumber domain of interest. The work presented in this paper is a generalization of the design method presented in [15] to general domains (for example ellipsoidal or cubical arrays). We discuss stability issues for sound field interpolation, and present some examples of more general measurement arrays, including a discussion on how to impose constraints on the design in order to deal with practical issues for building microphone arrays.

The paper is organized as follows: Section II gives a brief introduction into the approximation of wave fields and describes the microphone array performance measure applied in this study. In Section III, we first discuss the stability of sound field interpolation with spherical and non-spherical microphone arrays and then how the choice of a basis influences the stability of sound field representation. Several possibilities for designing microphone arrays are introduced in Section IV. In this section we discuss both the sampling on the domain's surface and how some interior points stabilize the array around unstable frequencies (e.g., Bessel nulls in the particular case of a spherical array). In Section V, the performance of spherical and ellipsoidal microphone arrays is compared using numerical simulations. The results highlight the effectiveness and flexibility of our method. However, when applying the proposed optimization method to spherical microphone arrays the interior sampling points are spread over the entire inner volume, which in practice may lead to problems with constructing the array. In Section VI, we therefore introduce a modified optimization method for determining the interior sampling positions that allows to include sampling constraints. Applying this method to three example arrays (a double sphere, a mixed sphere, and a spindle torus array; all with additional interior sampling points) shows how practical constraints are included into optimized spatial sampling, and how this improves the robustness and simplifies the practical implementation of these arrays. Concluding remarks are given in Section VII. The code to reproduce the results of this paper is available at http://gilleschardon.fr/jstsp_array.

\section{Preliminaries}

\section{A. Performance measures}

Microphone arrays can be used for a wide variety of tasks, e.g., sound source localization, beamforming, and sound field analysis. Various metrics can be applied to measure the performance of a microphone array, such as the white noise gain, the directivity index, and the condition number of the estimation of the spherical harmonics series expansion coefficients (see e.g. [16] for further quality metrics). In a previous study [15], we compared the performance of different open spherical arrays with respect to the interpolation error, the condition number, and the white noise gain.

In this work, we will use the interpolation error of the sound field inside the volume of the array as the main performance measure. This error is defined as the $L_{2}$ norm of the difference between the actual sound field $p$ and its interpolation $\hat{p}$ obtained from a finite number of measurements:

$$
\|p-\hat{p}\|=\int_{\Omega}|p-\hat{p}|^{2}
$$

For numerical integration adequate quadrature rules are applied. In this article, the sound pressure $\hat{p}$ is approximated in the domain of interest $\Omega$ by spherical harmonics approximations (cf. equation (3)); the spherical harmonics expansion coefficients were determined by least-squares estimation. While interpolation of the sound field is not always the goal of microphone array processing, the justification of the use of this measure for the design of microphone arrays is threefold: (i) the interpolation error is easy to estimate, (ii) theoretical results are available and can be used to guide the design of an array, and, what is even more important, (iii) the interpolation error can be used to represent any other performance measure. A microphone array, which is able to interpolate the sound field in its volume with a low number of microphones and a small interpolation error, is able to simulate any other microphone array included in the same volume, and thus inherits its performance.

\section{B. Approximation of acoustical fields}

Moiola et al. [17] rigorously studied the approximation of acoustical fields in the harmonic regime (or, more generally, of solutions to the Helmholtz equation). The main result of these studies is that a general solution $u$ to the Helmholtz equation

$$
\Delta u+k^{2} u=0
$$

in a star-convex domain $\Omega$ (i.e. there exists a point $O$ such that any point in the domain $\Omega$ can be linked to $O$ by a segment included in the domain) can be approximated by a linear combination of spherical harmonics

$$
u \approx \sum_{l=0}^{L} \sum_{m=-l}^{l} \alpha_{l m}^{L} j_{l}(k r) Y_{l m}(\theta, \phi)
$$


in spherical coordinates $(r, \theta, \phi)$, where $j_{l}$ is the $l$-th spherical Bessel function and $Y_{l m}$ are the spherical harmonics. Alternatively, the solutions can be as well approximated by a linear combination of plane waves

$$
u \approx \sum_{j=1}^{J} \beta_{j}^{J} \exp \left(i \vec{k}_{j} \cdot \vec{x}\right),
$$

where the wavevectors $\vec{k}_{j}$ are sampled on the sphere of radius $k$ in the wavenumber space. The convergence rate of those approximations depends on the smoothness of $u$. In most applications, $u$ is smooth and the convergence is exponential [18].

In the harmonic regime, the pressure field is a solution to the Helmholtz equation (2). It is thus possible to use the above introduced schemes to approximate sound fields in general convex domains.

Note that the expansion coefficients $\alpha_{l m}^{L}$ and $\beta_{j}^{J}$ depend on the order of approximation $L$ and $J$, respectively. A spherical harmonics series expansion of the acoustical field does not necessarily exist for general domains. A sphere centered at the origin is the only domain for which it is guaranteed that all acoustical fields can be represented as a series of spherical harmonics. For other domains, the sound field can only be approximated by finite sums of spherical harmonics, with an approximation error that tends to zero as the approximation order tends to infinity. This, however, has no consequences for the task at hand, and spherical harmonics can be safely used to interpolate the sound field in general star-convex domains.

\section{STABILITY OF SOUND FIELD INTERPOLATION}

In this section, we study the interpolation of a sound field $p$ from a finite number of punctual samples (i.e. pressure microphones) in a volume $\Omega$. The interpolation is obtained by least-squares estimation of the coefficients of a finitedimensional approximation $\hat{p}$ of the sound field, using plane waves, spherical harmonics, or other families of functions.

Stability is guaranteed when the interpolation error $\|p-\hat{p}\|$ is of the same order as the best approximation error $\|p-\tilde{p}\|$, where $\tilde{p}$ is the best approximation of $p$ in the chosen finite dimensional space. In general, having more measurements than degrees of freedom is not sufficient to ensure the stability of the interpolation (cf. Runge phenomenon [19]).

A popular way to capture sound fields is to sample them on the boundary of a volume $\Omega$, usually a sphere, and then interpolate the field inside the volume. It is well-known that this method fails at some frequencies. This can be formalized by considering the operator $W_{k}$ that maps the functions defined on the boundary $\partial \Omega$ to solutions to the Helmholtz equation with wavenumber $k$ in the domain $\Omega$. The norm of $W_{k}: L_{2}(\partial \Omega) \rightarrow L_{2}(\Omega)$ as an operator from $L_{2}(\partial \Omega)$ to $L_{2}(\Omega)$ can be estimated and is, for example, depicted for the sphere in Fig. 1. The norm of $W_{k}$ is close to infinity near the eigenfrequencies of the volume with Dirichlet boundary conditions. This means that a small error on the boundary $\Omega$ (e.g. due to measurement noise or interpolation errors) is amplified in the volume $\Omega$. It is important to note that this is independent of the particular sampling scheme on the surface

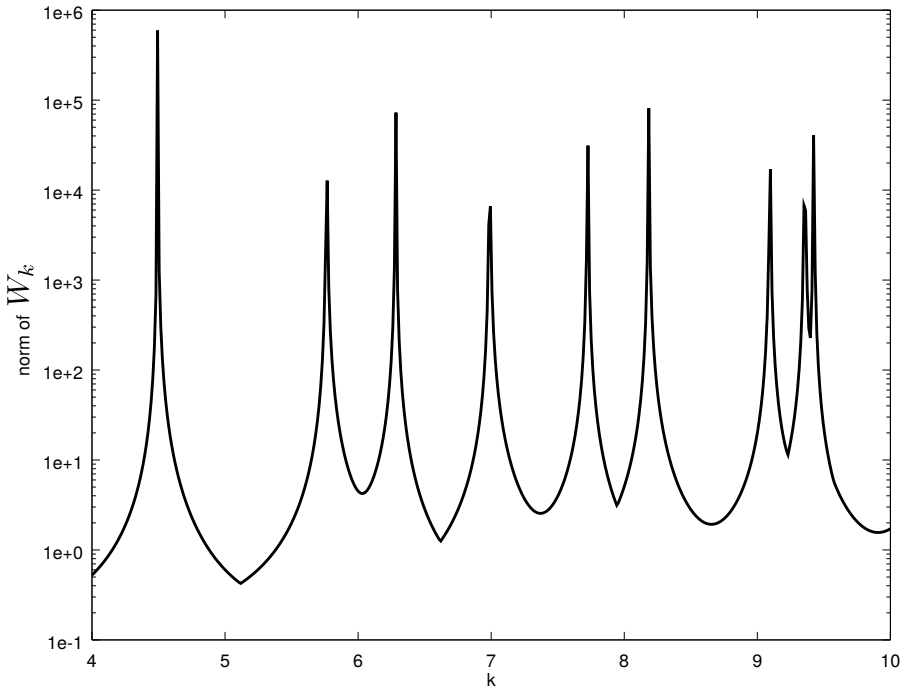

Fig. 1. Norm of the operator $W_{k}: L_{2}(\partial \Omega) \rightarrow L_{2}(\Omega)$ for the sphere of radius 1 . This norm is close to infinity near the eigenfrequencies of the volume and makes the interpolation of the sound field unstable.

of the volume and the approximation scheme used for the interpolation, and that the unstable frequencies only depend on the shape of the volume.

Various methods have been proposed to deal with these instabilities. The double sphere array, for example, uses two spheres with different radii to ensure that at least one of the spherical arrays is able to interpolate the sound field (cf. [6], [7], [8]). The main disadvantage of the double sphere array is that it needs at least twice the number of sampling points as the degrees of freedom. Another approach, proposed by Rafaely [9], guarantees a stable estimation of the approximation coefficients by using some additional measurements inside the sphere. However, this promising method is lacking in some aspects. The number of necessary interior sampling points has not been studied, and the microphone positions are determined with computationally intensive methods. This method is also limited to spherical arrays.

In this paper, we present a novel and computationally efficient method (in terms of number of required sampling points and of computational complexity for estimating the sampling positions) for increasing the stability around the eigenfrequencies of a general domain. The proposed optimization method builds upon the findings of Cohen et al. [20] that, given a basis for the finite dimensional approximation of the sound field and a sampling scheme (i.e. a distribution of samples inside the considered domain $\Omega$ ), allow the estimation of the minimal number $n$ of measurements necessary for a stable interpolation. Given a probability density $\mu$ - which is used to draw the measurement points in the domain $\Omega-$ and an orthogonal basis $\left(e_{i}\right)$ (with respect to $\mu$ ) for the finitedimensional spaces $E_{m}$ of dimension $m$, in which we search for an approximation of $p-$ e.g., the space spanned by the spherical harmonics up to order $L$, with $m=(L+1)^{2}-$ we 
can compute the quantity

$$
K(m)=\max _{x \in \Omega} \sum_{j=1}^{m}\left|e_{j}(x)\right|^{2} .
$$

Theorem 2 of [20] shows that if for a given constant $C$ smaller than $(1-\log 2) / 2 \approx 0.153$ the number of measurements $n$ is such that $K(m)<C n / \log n$, then with a high probability depending on the choice of $C$ the estimation error is of the same order as the best approximation error. The smaller the constant $C$ the higher is the probability. In practice, $K(m)$ measurements are sufficient for a stable interpolation. A good sampling scheme yields a value of $K(m)$ close to its lower bound $m$.

We now give numerical estimations of $K(m)$ for various sampling schemes and domain shapes. The results obtained in this section are not constructive, but give insight into the performance of various sampling schemes, which help to design microphone arrays. This will be demonstrated in the next sections.

\section{A. Spherical microphone arrays}

Chardon et al. (cf. [21], [22]) studied the particular case of the sphere and showed that for a uniform density of samples inside the ball the number of needed measurements to achieve stability is in the order of $L^{3}$, where $L$ is the maximal order of the spherical harmonics and corresponds to $(L+1)^{2}$ degrees of freedom. If only a proportion $\alpha \in] 0,1[$ of samples is used on the surface of the sphere, while the rest of the samples remains inside the ball, the minimal number of measurements is equivalent to $L^{2} / \alpha$.

Fig. 2 shows the values of $K(m)$ computed for $k r=3$ and three different distributions:

- a distribution uniform in angle and radius,

- a distribution uniform in the ball,

- a mixed distribution (we choose here $\alpha=0.8$ ).

It can be seen from Fig. 2, that the most efficient sampling scheme for $L>k r=3$ (i.e. when the spherical harmonics approximation becomes relevant) is a mixed distribution with most of the measurements on the border of the domain. In this case the values of $K(m)$ are close to the lower bound $(L+1)^{2}$ for an increasing $L$. In other words, using more measurements near the border of the domain than inside the domain allows for a lower total number of sampling points.

\section{B. Non-spherical arrays}

In this section, we study two different non-spherical arrays, the ellipsoid and the cube. Following the results obtained for the sphere, we restrict the analysis to mixed distributions with most of the measurements on the boundary of the domain. The interior measurements are drawn from a uniform distribution inside the domain. It will be further shown, that it is of particular importance to carefully choose the distribution of measurements on the border of the domain. The following sample distributions will be compared:

- a uniform density of samples on the surface of the domain;

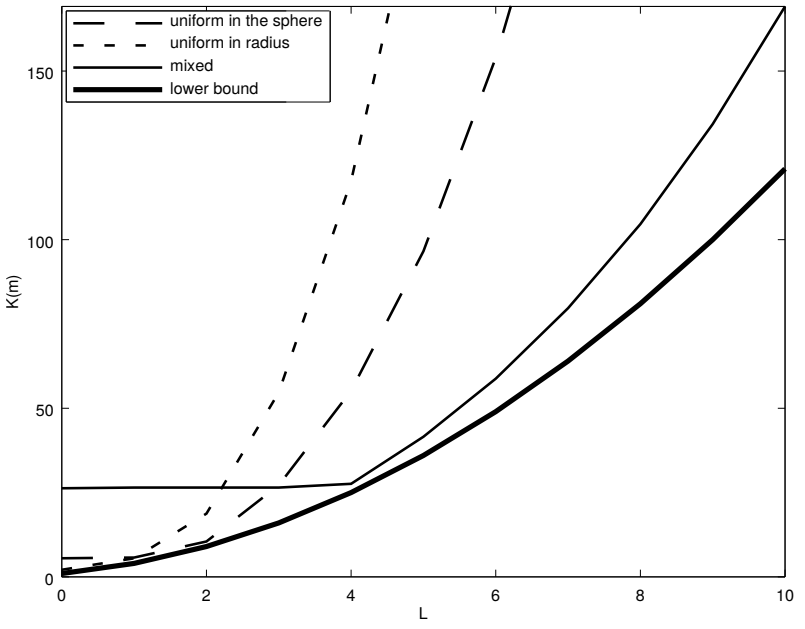

Fig. 2. Minimal number of measurements $K(m)$ for the sphere as a function of the order of the spherical harmonics approximation $L$.

- samples obtained by the projection of a uniform distribution on the sphere to the border of the domain (as has been suggested in [12]);

- a particular distribution, which is adapted to each of the considered domains.

The particular distribution for the ellipsoid is obtained by stretching the sphere to the ellipsoid, as depicted in Fig. 3. For the cube the density on the boundary, e.g., on the faces parallel to the $x y$-plane, has the form

$$
\mu=\frac{1}{Z} \frac{1}{\sqrt{1-x^{2}} \sqrt{1-y^{2}}},
$$

where $Z$ is a constant such that the total weight is 1 . Similar expressions can be derived for the other faces of the cube. It should be further noted that these distributions result in more points far from the center, while the projected uniform distribution gets denser close to the center. Fig. 3 illustrates this behavior for the ellipsoid.

Figs. 4 and 5 show the values of $K(m)$ as a function of $L$ for different sample distributions with $\alpha=0.8$ for both the ellipsoid (i.e. an oblate ellipsoid of revolution with an aspect ratio of $1 / 2$ ) and the cube, respectively. It can be seen, that the projected uniform density performs worst (apart from the uniform density in the volume), and that the proposed particular distributions perform close to the lower bound. The simulation results further show that projecting a sample distribution from the sphere to the measurement surface is, in general, not a good choice. The sample distribution should be adapted to the shape of the domain. A method for designing such a well adapted sampling scheme for arbitrary surfaces is described in Sec. IV-A.

\section{Influence of the approximation scheme}

In general, the minimal number of sampling points does not only depend on the choice of the sampling density, but also on the basis functions that are used to approximate the acoustical field (e.g., plane waves, spherical harmonics, spheroidal harmonics, fundamental solutions, etc.). However, numerical 
(a)
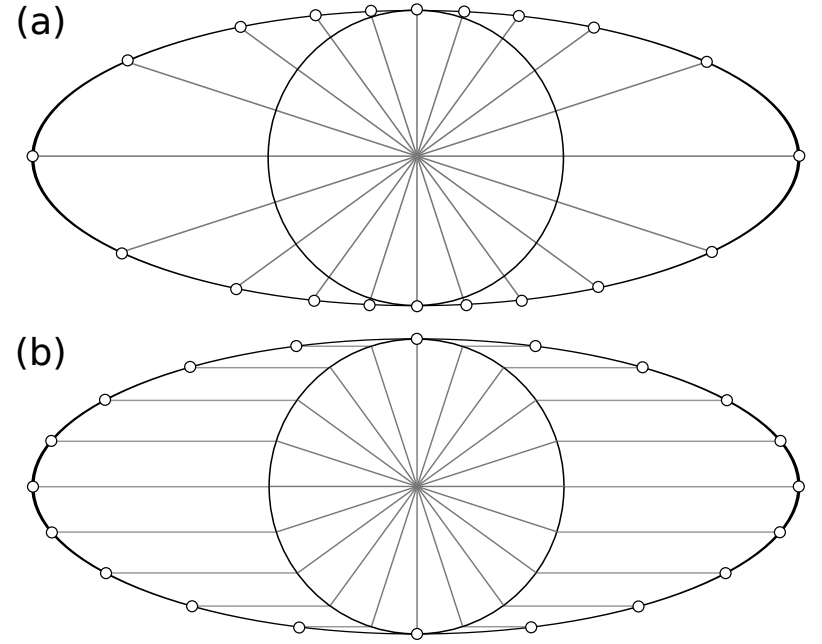

Fig. 3. Case of the ellipsoid: (a) projected uniform distribution of the sphere, (b) stretched distribution.

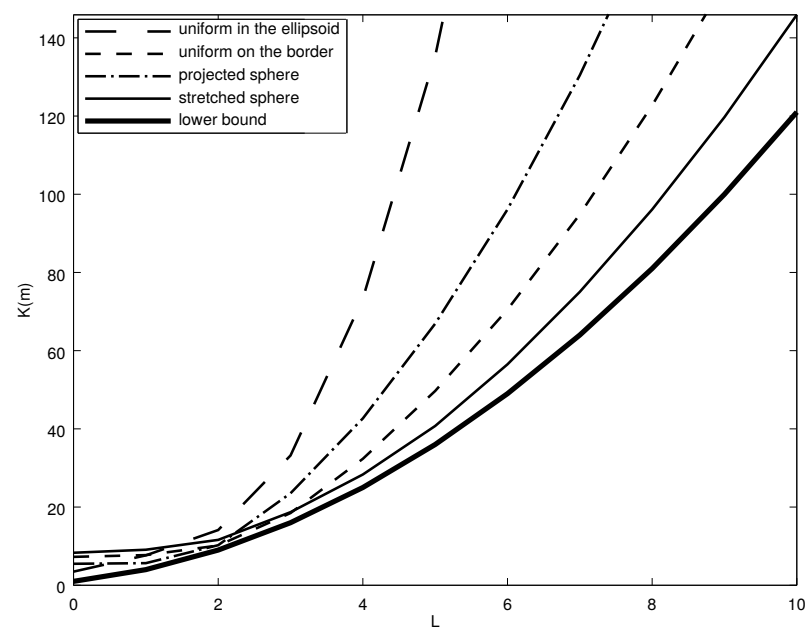

Fig. 4. Minimal number of measurements $K(m)$ for the ellipsoid (oblate with aspect ratio 0.5 ) as a function of the order of the spherical harmonics approximation $L$.

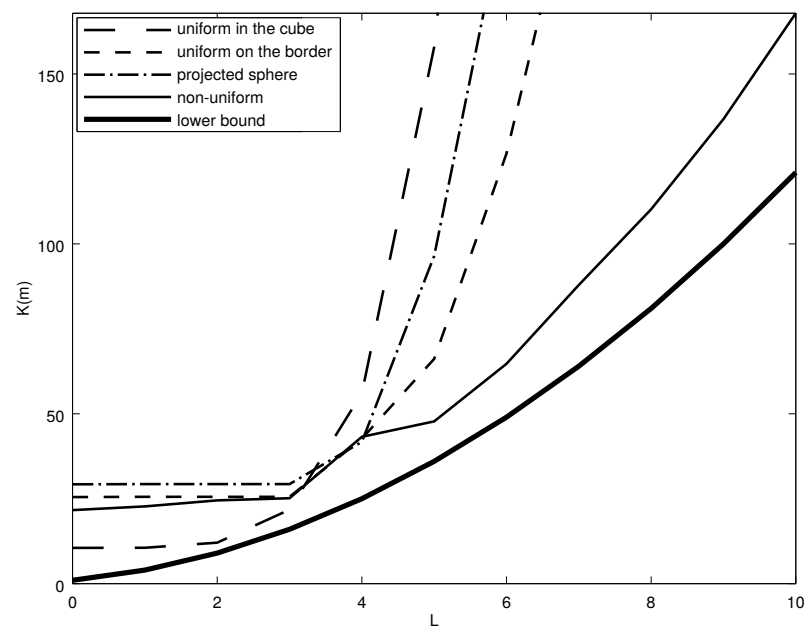

Fig. 5. Minimal number of measurements $K(m)$ for the cube as a function of the order of the spherical harmonics approximation $L$.

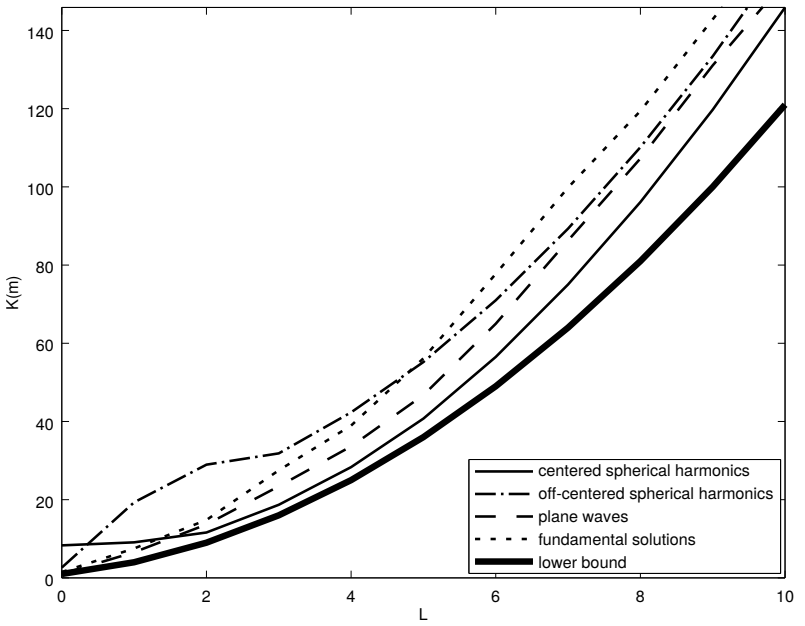

Fig. 6. Minimal number of measurements $K(m)$ as a function of the approximation order $L$, plotted for different approximation schemes for a stretched sample density on the ellipsoid.

tests have shown that for large $m$ the behavior of $K(m)$ does not depend on the choice of the basis functions. Fig. 6 demonstrates this for the ellipsoid using spherical harmonics centered at the origin of the ellipsoid, spherical harmonics centered at $(1,1,1)$ (outside of the domain), plane waves, and fundamental solutions. The plane waves are chosen with wavevectors on a sphere of radius $k=3$ in the wavenumber space, sampled by the Sloan-Womersley points [23], [24]. For the fundamental solutions the sources were on a sphere with radius $r_{s}=2$, applying the same sampling grid. $K(m)$ is plotted for a stretched distribution with $\alpha=0.8$. Its values remain close to the lower bound for the different approximation schemes. While this property has yet to be proven theoretically, it suggests that the stability is independent of the choice of the approximation scheme. For this reason, only spherical harmonics will be considered in the remainder of this paper.

In summary, the following conclusions can be drawn: To ensure stability of interpolation with a minimum number of measurements, only a few inside-the-domain measurements are needed at the modal frequencies of the domain of interest. Uniform distributions and uniform distributions on the sphere projected on the boundary of the domain of interest are in general not efficient. Two examples, the ellipsoid and the cube, are given to illustrate this behavior. For these two domains, a particular non-uniform distribution was shown to give a value of $K(m)$ close to its lower bound. Yet it is still an open question how to design an optimal distribution for general domain shapes.

The following sections discuss practical methods for the design of robust microphone arrays, which are based on these results.

\section{DESIGN OF A MICROPHONE ARRAY}

In this section, we introduce a design method for robust open microphone arrays, which is based on the theoretical findings of the previous sections. The proposed method divides the design of the microphone array into two sub-tasks: (i) 
the design of a sampling grid on the border of the volume, and (ii) the choice of sampling points inside the volume that stabilize the interpolation at the eigenfrequencies of the considered domain. For sampling the surface of arbitrary starshaped domains, we propose using the magic points method [25], which is briefly described in the next section.

\section{A. Magic points}

Given a family of linearly independent functions for approximating a particular space of functions, the magic points method can be used to derive a set of sampling points. This method was originally developed for numerical analysis. We here introduce the magic points as a design method for sensor arrays.

Given a vector space of functions of dimension $m$, this method identifies $m$ sample points that can be used for a stable interpolation of the functions. In its simplest form, the magic points can be computed with an iterative process. Given a set $\left(e_{i}\right)$ of $m$ linearly independent functions, the $j$-th measurement point is obtained by the following maximization procedure:

$$
x_{j}=\underset{x \in \Omega}{\operatorname{argmax}} \tilde{e}_{j},
$$

where $\tilde{e}_{j}$ is a linear combination of the first $j$ functions $e_{i}$ with zero values at the first $(j-1)$ identified points $x_{i}$. That is $\tilde{e}_{j}(x)=e_{j}(x)-\sum_{i=1}^{j-1} \alpha_{i}^{j} e_{i}(x)$, where the $\alpha_{i}^{j}$ are chosen such that $\tilde{e}_{j}\left(x_{i}\right)=0$ for $i<j$. For a more detailed description the authors refer to [25].

For a robust microphone array, a sampling scheme should allow for a stable interpolation over a large frequency band. For this reason, we use a slightly modified version of the magic points algorithm. Let us consider multiple families $\left(e_{i}^{n}\right)$, which are associated to the wavenumbers $k_{n}$. Following Moiola et al. [17], we choose the sets of functions $j_{l}\left(k_{n} r\right) Y_{l m}(\theta, \phi)$ for wavenumbers $k_{n}$ and orders up to $L$ to approximate the acoustical fields. Then the measurement points can be identified by the following optimization problem:

$$
x_{j}=\underset{x \in \Omega}{\operatorname{argmax}} \min _{n} \tilde{e}_{j}^{n},
$$

where $\tilde{e}_{j}^{n}$ is defined as in (6). Other variants, with possibly better interpolation properties exist. However, the simplified method used here yields good interpolation results, as will be shown below.

While designing an array with this method is straightforward, the magic points method does not explicitly take the theoretical results on the interpolation of acoustical fields into account. A more sophisticated method is now introduced. Based on the theoretical results, the design of the array is divided into two parts: a sub-array of microphones on the boundary of the domain, and a smaller sub-array of microphones inside the domain.

\section{B. Sampling the surface}

As shown in Sec. III-B, a particular distribution of measurements on the border of the considered volume has to be used to guarantee a stable interpolation with only a small number of measurements. For some special cases, such as the sphere or the ellipsoid, this sample distribution can be easily found.

For the sphere, we use the optimal sets of sampling points given by Sloan and Womersley (cf. [23], [24]). These point sets can interpolate a function, which is defined on the sphere, with as many sampling nodes as spherical harmonics used to represent the function. This special case of sampling is often referred to as 'critical sampling' on the sphere. With reference to the results given in Sec. III-B, the distribution for the ellipsoid is obtained by stretching the sample distribution on the sphere to the ellipsoid.

For generic measurement surfaces, the sample distribution is derived from the magic points method. The optimization problem in (7) is used, with the slight modification that the considered domain is restricted to the boundary $\partial \Omega$ of the domain $\Omega$. Note that the authors do not claim that the magic points method yields an optimal set of sampling points. Nevertheless, this method can be easily applied to the discussed problems and, as will be shown in Secs. V-A and V-B, provides adequate sampling grids, e.g., for spherical, ellipsoidal, and spindle torus arrays.

\section{Choice of the interior points}

The measurement points inside the considered volume have to be carefully chosen. If a measurement point lies on the nodal surface of a particular eigenmode, it cannot be used to stabilize the interpolation for this frequency.

In the following, we introduce a method for determining the optimal positions of the interior sampling points. We first consider the simpler case, i.e. when the eigenfrequencies are not degenerate. In order to be estimated accurately, an eigenmode should be measured at a point where it has the largest amplitude. When considering a frequency band with more than one eigenfrequency, it is highly unlikely that all the eigenmodes have the same maximum. Therefore, we sample the sound field at that point for which the minimal amplitude of the modes $p_{n}$ is the largest in the considered domain:

$$
x=\underset{x \in \Omega}{\operatorname{argmax}} \min _{n}\left|p_{n}(x)\right| .
$$

At this particular point the values of the modes are guaranteed to be nonzero (because the nodal surfaces have measure zero and so has their union), and thus allow to estimate the eigenmodes using only one point. Note that while the amplitudes of several modes have to be estimated, they do not occur at the same frequency, using only one measurement for this estimation is therefore possible.

For certain geometries (e.g., the sphere), most of the eigenfrequencies are degenerate. In this case, it is impossible to estimate the modes with only one microphone; at least $M$ microphones, where $M$ is the largest multiplicity of the eigenfrequencies, are needed. We propose to estimate these $M$ points iteratively. The first point is found as the solution of the following optimization procedure:

$$
x_{1}=\underset{x \in B}{\operatorname{argmax}} \min _{j} \max _{\substack{p \in E_{j} \\\|p\|=1}}|p(x)|,
$$


where $E_{n}$ are the eigenspaces associated to the eigenfrequencies in the considered frequency range.

The next point is found by considering the subspaces $E_{j}^{1}$ of the eigenspaces of dimension more than 1 , where the functions are zero at the first identified measurement point:

$$
x_{2}=\underset{x \in B}{\operatorname{argmax}} \min _{j} \max _{\substack{p \in E_{j} \\\|p\|=1 \\ p\left(x_{1}\right)=0}}|p(x)| .
$$

Then the $n$-th point is found by using the spaces $E_{j}^{n-1}$ of the eigenspaces of dimension more than $n-1$, where the functions are 0 at the first $n-1$ identified measurement points.

The measurement points are thus found by solving an iterative sequence of minimization procedures

$$
x_{i}=\underset{x \in B}{\operatorname{argmax}} \min _{j} \max _{\begin{array}{c}
p \in E_{j} \\
\|p\|=1 \\
\left(p\left(x_{j}\right)=0\right)_{0<j<i}
\end{array}}|p(x)| .
$$

Note that if only one eigenspace is considered, this algorithm reduces to a variant of the magic points method.

\section{Simulation RESUlTS}

This section discusses the numerical simulation results and shows that the proposed design methods yield efficient and robust microphone arrays, i.e. microphone arrays that are able to interpolate the acoustical field in their volume with a limited number of sampling nodes, that is comparable to the number of degrees of freedom used for the approximation of the sound field. As already pointed out in Sec. II, accurate interpolation within the array volume most likely results in a good estimation of the spherical harmonics expansion coefficients, the white noise gain, and other performance measures.

We measure the array performance using the interpolation error of plane waves inside the volume of the array. For all numerical experiments, measurements are simulated with white Gaussian noise signals and a signal-to-noise ratio (SNR) of $40 \mathrm{~dB}$. The results are then averaged over 40 random directions of arrival of the respective plane waves. The error is normalized with respect to the total energy of the sound field in the volume. The chosen value for the SNR makes it possible to highlight the two ranges of frequencies where the interpolation error is limited by the noise level and the approximation error, respectively.

\section{A. Spherical arrays}

In a previous study [15], we compared the performance of different open spherical arrays with respect to the interpolation error, the condition number, and the white noise gain. In the following, we briefly summarize the results with respect to the interpolation error and extend the study to two new sampling grids constructed using the magic points method. For all arrays the basis for approximating the sound field is given by the product of the spherical harmonics with the spherical Bessel functions, see (3). Fig. 7 depicts the interpolation error for the following array configurations and for $k \in] 0.5,9[$ :
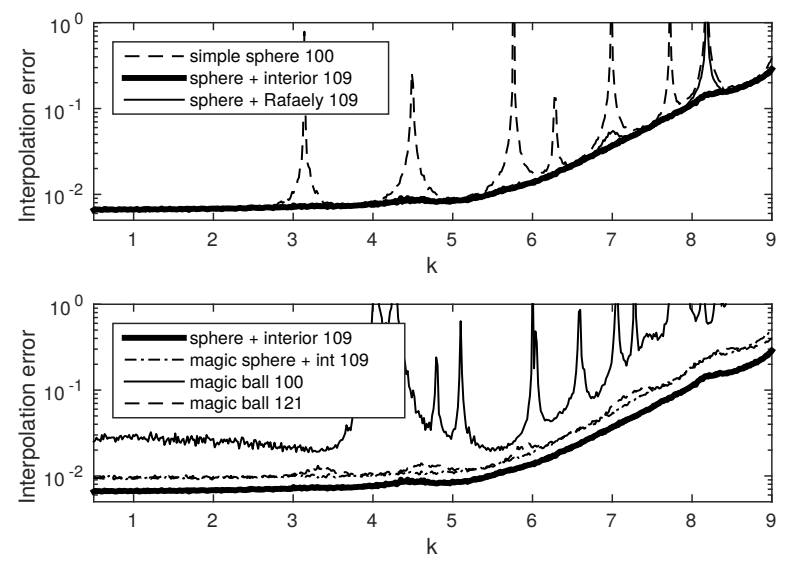

Fig. 7. Interpolation error for the spherical array with different sampling schemes described in Section V-A.

- Simple sphere: Open sphere array with 100 sampling nodes on the sphere that correspond to the SloanWomersley 'hyperinterpolation' grid (see [23], [24]) for an array order of 9. No interior points are used.

- Sphere plus interior: Open sphere array that uses the same sampling scheme on the sphere as the 'simple sphere' array, but with additional 9 points (i.e. the maximal multiplicity of eigenmodes in the considered frequency range) inside the sphere. The interior sampling nodes are determined by the algorithm described in Sec. IV-C.

- Magic sphere plus interior: Open sphere array with 100 magic points on the sphere and 9 additional points (i.e. the maximal multiplicity of eigenmodes in the considered frequency range) inside the sphere. The interior sampling nodes are determined by the algorithm described in Sec. IV-C.

- Sphere plus Rafaely: Open sphere array that uses the same sampling scheme on the sphere as the 'simple sphere' array. The 9 additional sampling nodes inside the sphere are determined by the method given in [9].

- Magic points in the ball: Magic points sampling is used on the whole ball, where the maximum order of the spherical harmonics basis is set to $N=9$ and $N=10$ with 100 and 121 sampling nodes, respectively.

Fig. 7 clearly shows the stability improvements around the Bessel nulls due to the interior points. The curve for the array without interior points has peaks at the eigenfrequencies of the sphere. These peaks can be mostly suppressed by adding measurement points inside the sphere, computed with the approach proposed in this paper. For the proposed array (sphere plus interior), the interpolation error for $k<5$ results from the noisy measurements; for $k>5$, the interpolation error is limited by the approximation error of the 9th-order spherical harmonics expansion of the sound field. A higher SNR would result in lower interpolation errors for $k<5$. Lower interpolation errors for $k>5$ can only be achieved by using higher order spherical harmonics and hence more microphones. 
Comparing the proposed optimization method with Rafaely's approach [9] for determining the interior sampling nodes, the latter shows one prominent peak at $k=8.18$. This peak is most likely caused by the uniform sampling in angle used for the interior points. Replacing the hyperinterpolation sampling grid on the sphere by magic points (and using the interior points derived by our approach) results in a slightly increased interpolation error. The behavior of the error can be explained as follows: the magic points algorithm solves the sampling problem iteratively and is not expected to perform as good as the highly optimized hyperinterpolation approximation on the sphere. The main advantage of using the magic points method is its simple construction algorithm, which makes it suitable for the use with arbitrary array geometries, for which optimal sampling grids have not yet been investigated. The magic points sampling grid for the ball with 100 nodes does not result in a robust array; however, when using 121 sampling nodes it performs almost as good as the proposed method. The simplicity of the magic points method thus makes it a good alternative to the proposed method when the computation of the eigenfrequencies and eigenmodes of a domain is too complex.

\section{B. Ellipsoidal arrays}

As already mentioned, the proposed method for determining the interior sampling nodes is not limited to spherical arrays and can be used for any arbitrary star shaped domain. In a second example, we therefore apply this method to an ellipsoidal array, where the semi-axes are given by $a=1.0, b=0.8$ and $c=0.5$. The eigenfrequencies and eigenmodes are determined using the method of particular solutions (cf. [26], [27]). Similar to the spherical arrays in the previous section, we use 100 sampling points on the surface of the ellipsoid. However, only one additional sampling point inside the ellipsoid is needed, since all eigenfrequencies in the observed wavenumber domain are simple. In Fig. 8 the interpolation error for the following arrays is given:

- Stretched distribution: Ellipsoid with 100 sampling nodes on the surface that correspond to the SloanWomersley sampling on the sphere stretched to the ellipsoid (see Fig 3b). No interior points are used.

- Stretched plus interior: Ellipsoid with 100 sampling nodes as for the 'stretched distribution', but with one additional single node inside the ellipsoid. This sampling point is determined by the algorithm described in Sec. IV-C.

- Projected plus interior: The nodes on the ellipsoid are determined by a radial projection of the SloanWomersley sampling grid on the sphere (see Fig. 3a). The interior point is determined by the algorithm described in Sec. IV-C.

- Magic points plus interior: The surface of the ellipsoid is sampled using 100 magic points. The one additional interior node is determined by the algorithm described in Sec. IV-C.

- Magic points in the ellipsoid: Magic points in the entire ellipsoid. The maximum order of the spherical harmonics
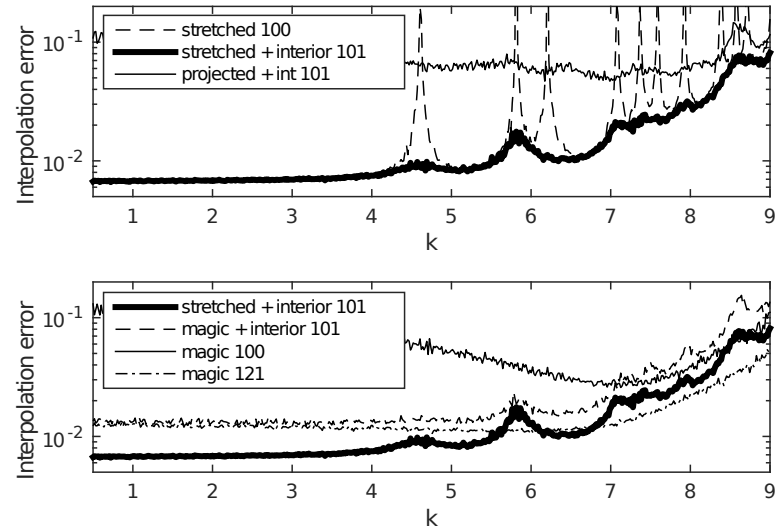

Fig. 8. Interpolation error for the ellipsoidal array with $a=1.0, b=0.8$, and $c=0.5$.

is set to $N=9$ and $N=10$ with 100 and 121 sampling nodes, respectively.

For the error a similar behavior as for the spherical arrays can be observed. Without the additional interior point, the interpolation error has several peaks. For the ellipsoid used in this example, all eigenspaces in the observed wave number domain have the dimension one. The stability of the array can be improved within a large frequency range by simply adding one single node inside the array. It is important to note that the different methods for estimating the sampling nodes on the ellipsoid have a strong effect on the performance of the array. The error for the radial projection of the Sloan-Womersley grid is much larger than the error for the stretched grid. In general, the 'stretched plus interior' grid with 101 sampling nodes performs very similarly to the magic points array with 121 sampling nodes, i.e. where the nodes have been determined using the magic points algorithm. The latter array provides lower interpolation errors for $k>5.5$, which is not surprising considering the higher number of sampling nodes. Using the magic points method with only 100 sampling nodes (i.e. the number of degrees of freedom) results in a poor interpolation.

As observed in the spherical case, the combination of magic points on the surface plus interior points determined by the proposed algorithm yields a slightly higher interpolation error than the stretched Sloan-Womersley grid with interior points. Nevertheless, the results presented in Fig. 8 indicate that for general star shaped domains the combination of magic points on the surface of the array with additional interior points determined by the proposed approach provides good sampling points.

\section{OPTIMIZING MICROPHONE ARRAYS WITH PRACTICAL CONSTRAINTS}

The results given in the previous sections do not take the practical implementation of a microphone array into account. In particular, the interior points for the sphere are distributed over the volume of the array without any regular structure. This makes it often difficult to build the array structure. In this section, we provide three examples that demonstrate how 
to include practical constraints in the optimization of the microphone array. The first two examples show the design of spherical arrays, where the interior microphone positions are restricted to an inner sphere (i.e. an open as well as a closed, sound hard sphere as investigated in [8]). The third example optimizes a spindle torus array, that can be implemented with a simple scanning array as shown in [12].

\section{A. The double sphere}

The classical double sphere array consists of two concentric spheres with similar sampling grids, which are determined by the array order. This sampling method is inefficient as the number of sampling nodes has to be at least twice the number of degrees of freedom (i.e. twice the number of spherical harmonics used for representing the sound field). We know from Sec. V that only a few interior microphones are required to improve the robustness of the array at the eigenfrequencies. The design of double sphere arrays can be optimized using the method proposed in this article (see Sec. IV). To guarantee that the interior sampling nodes lie on a sphere, the optimization method is slightly modified and consists of two steps. Firstly, the radius $\rho$ of the inner sphere is determined by the first point computed with (11). Secondly, the following points are obtained by the same optimization problem with the choice of $x_{i}$ restricted to the sphere of radius $\rho$.

Fig. 9 shows the simulation results for a 9th-order spherical array for frequencies $k \in[0.5,9]$. The simulation uses 100 Sloan-Womersley sampling nodes on the sphere, and 9 additional sampling nodes inside the sphere; the interior measurement points are restricted to an inner sphere with radius 0.69 . We compare the interpolation error of the optimized double sphere array to (i) a simple sphere, (ii) the optimized spherical array given in Sec. V, (iii) a double sphere array with the same radius for the inner sphere but a 2nd-order Sloan-Womersley sampling grid on the inner sphere (which results in the same number of required microphones than for the optimized double sphere), and (iv) a double sphere array, with an inner radius $\rho=1 /\left(1+\frac{\pi}{2 k_{\max }}\right) \approx 0.85$, as suggested in [6]. The simulation results show that the optimized double sphere is slightly less stable than the unconstrained open sphere array, but also has a low interpolation error at the eigenfrequencies of the ball. It can be further seen that the array with a quasi-regular sampling on the inner sphere becomes unstable at $k \approx 8.17$. This behavior has been observed for the open sphere array that was optimized with Rafaely's method (see Sec. V-A). We can conclude that it is, in general, better to use a non-regular sampling scheme for the interior points. Using the inner radius suggested in [6] the estimation becomes unstable near the lowfrequency modes. This can be explained as follows. When the inner sphere is too close to the outer sphere, the low-frequency eigenmodes have very low values on the inner sphere and thus cannot be accurately estimated.

\section{B. The mixed spheres}

Jin et al. [8] proposed a double sphere array that combines an open outer sphere with a closed and sound hard inner sphere. Our optimization method can be adapted to this case,
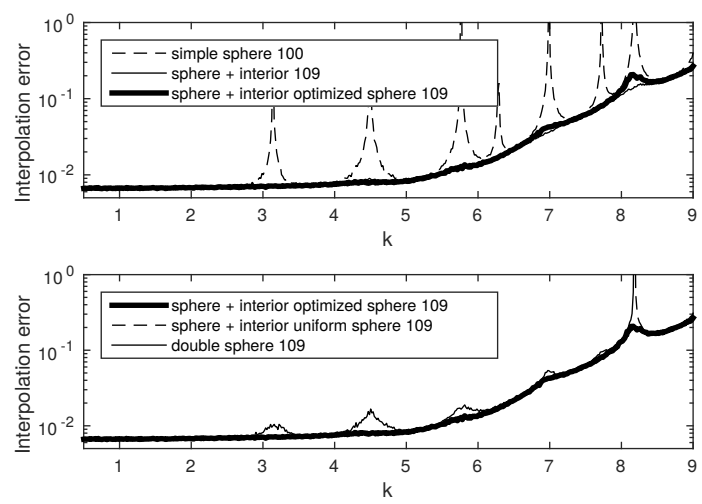

Fig. 9. Interpolation error for the double sphere, for $k \in[0.5,9]$, spherical harmonics approximation of order 9 and 109 measurements, for various microphone array designs.

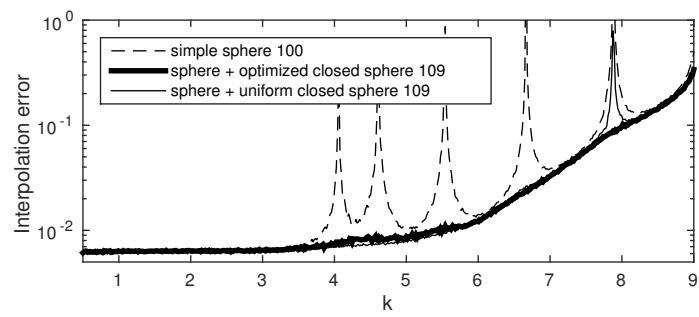

Fig. 10. Interpolation error for the mixed sphere, for $k \in[0.5,9]$, spherical harmonics approximation of order 9 and 109 measurements, for various microphone array designs. The radius of the inner rigid sphere is 0.5 .

as will be shown in the following. When using only the measurements on the outer sphere, the interpolation of the sound field becomes unstable at the eigenfrequencies of the domain that is limited by the two spheres, with Dirichlet boundary conditions on the outer sphere $S_{o}$ and Neumann boundary conditions on the inner sphere $S_{i}$. As the eigenmodes satisfy the Neumann boundary conditions on $S_{i}$, they are nonzero almost everywhere on this sphere. Therefore, pressure microphones can be used to stabilize the interpolation near the eigenfrequencies. The interior sampling nodes are found by the optimization problem in (11), with the additional constraint that they are restricted to $S_{i}$, and by using the eigenspaces for the appropriate domain and boundary conditions.

The interpolation error of an array with no interior points, the optimized array, and an array with a quasi-uniform sampling on the inner sphere is given in Fig. 10. As in the case of an open sphere, the obtained sampling on the inner sphere yields stability on the entire frequency range, while the quasiuniform sampling fails at $k \approx 7.88$.

\section{The spindle torus array}

Alon and Rafaely [12], introduced a non-spherical microphone array with a spindle torus sampling grid. The particular shape of this array can be easily implemented with a scanning microphone array, and includes points in the interior of the domain of the array. The sampling nodes were obtained by projecting the sampling grid of a sphere to the surface of the spindle torus. As we have shown in Sec. III the projection 

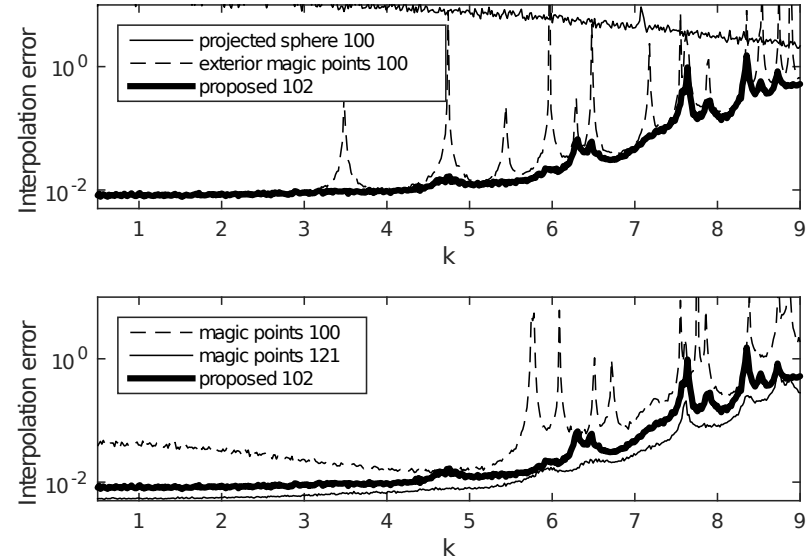

Fig. 11. Interpolation error for the spindle torus with radiuses $R=0.3$ and $r=0.7$, for $k \in[0.5,9]$, with approximation order $L=9$.

results in an inefficient sampling of the wave field. Therefore, we adapt the magic points method IV-A to the spindle torus array by restricting the possible interior sampling nodes to the surface of the torus (and do not sample the entire domain). The proposed method is also tested, by constraining the interior points to lie on the interior part of the torus. The sampling points inside the spindle torus stabilize the interpolation at the eigenfrequencies of the domain delimited by the outer part of the torus.

Fig. 11 depicts the numerical simulation results. The spindle torus is defined by the parametric equations

$$
\begin{cases}x=(R+r \sin \theta) \cos \phi & \phi \in[0,2 \pi[ \\ y=(R+r \sin \theta) \sin \phi & \theta \in[0,2 \pi[ \\ z=r \cos \theta & \end{cases}
$$

Simulations were computed for $R=0.3, r=0.7$, and a spherical harmonics approximation of order 9 (i.e. 100 degrees of freedom); the considered wavenumbers were between 0.5 and 9. The following arrays are compared:

- A quasi-uniform 9th-order sampling grid (i.e. 100 points) on the sphere projected on the spindle torus. Following the suggestions given in [12], half of the measurements are on the exterior surface, and the other half lie on the interior surface; the assignment of sampling nodes is random.

- Magic points on the spindle torus using spherical harmonics of order 9 (i.e. 100 points) and 10 (i.e. 121 points).

- Magic points that only consider points on the exterior surface of the spindle torus, with spherical harmonics of order 9 (i.e. 100 points).

- Proposed method: Magic points on the exterior surface of the torus plus 2 additional sampling points on the interior surface (i.e. a total of 102 points); the interior sampling nodes are obtained by the method introduced in Sec. IV-C.

Fig. 11 plots the interpolation error inside the torus for the five different arrays. When applying the spatial sampling method proposed by Alon and Rafaely the interpolation inside the array becomes unstable. With the magic points on the border of the domain we obtain less interpolation error, but the interpolation becomes unstable at the eigenfrequencies of the volume delimited by the exterior part of the spindle torus. This result clearly shows that using sampling nodes at only a few different radii is not sufficient to stabilize the interpolation of the sound field. Only interior points help to improve the robustness of the array. With the proposed method, we only use two additional sampling nodes inside the array (i.e. 102 microphones for an array of order $N=9$ ) to stabilize the interpolation at most of the eigenfrequencies and, as a result, obtain low interpolation errors for $k<7.5$. This is possible as the eigenfrequencies are at most double (i.e. the associated eigenspaces have a dimension of at most two). The array with 100 magic points on the spindle torus performs less good than the proposed array with 102 sampling nodes. When using 121 magic points the interpolation error is lower than that for the proposed array. However, the improved interpolation comes at a price of 20 additional microphones.

\section{CONCLUSION}

In this paper, we studied the analysis and the practical design of spatial microphone arrays. The analysis clearly shows that a stable interpolation of the sound field (or equivalently, a stable estimation of the spherical harmonics approximation coefficients) over a broad frequency range can only be guaranteed when using a limited number of sampling nodes inside the volume of the array. In the case of non-spherical arrays, a particular non-uniform distribution of measurements on the surface of the array is needed to increase the robustness of the array. It was further shown that projecting a uniform distribution on the sphere on the volume of interest is inefficient.

We introduced two methods for the practical design of spatial microphone arrays: (i) The first method is based on the magic points method, which was originally introduced as an algorithm for numerical analysis. To the best of the authors' knowledge, this is the first time that magic points are applied to the design of spatial sensor arrays. Magic points are easy to implement and have been shown to provide lower interpolation errors than other sampling grids previously introduced in literature. (ii) The second method is based on a theoretical analysis of the approximation of acoustical fields in the harmonic regime. This novel method allows for an accurate interpolation of the sound field with a number of microphones that is close to the number of degrees of freedom used for the approximation. In this respect it clearly outperforms the magic points method. The proposed method, however, requires the computation of the eigenfrequencies and eigenmodes of the domain of the array within the frequency band of interest, which is a non-trivial matter for general domain shapes.

It was further demonstrated that practical constraints can be considered in the optimization of an array, and that in some particular cases (e.g. for the ellipsoid with no symmetry), only a single interior measurement is needed to achieve stability over a large frequency band.

\section{ACKNOWLEDGMENT}

This research was supported by the Austrian Science Fund (FWF) Start Project FLAME (Frames and Linear Operators for 
Acoustical Modeling and Parameter Estimation; Y 551-N13), the French ANR CONTINT project SOR2 (Sample Orchestrator 2), and the S\&T cooperation project Amadeus AustriaFrance 2013-14, "Frame Theory for Sound Processing and Acoustic Holophony", FR 16/2013.

\section{REFERENCES}

[1] T. D. Abhayapala and D. B. Ward, "Theory and design of high order sound field microphones using spherical microphone array," in Acoustics, Speech and Signal Processing (ICASSP), IEEE International Conference on, 2002, pp. 1949-1952.

[2] B. N. Gover, J. G. Ryan, and M. R. Stinson, "Measurements of directional properties of reverberant sound fields in rooms using a spherical microphone array," The Journal of the Acoustical Society of America, vol. 116, no. 4, pp. 2138-2148, 2004.

[3] J. Meyer and G. W. Elko, "A highly scalable spherical microphone array based on an orthonormal decomposition of the soundfield," in Acoustics, Speech and Signal Processing (ICASSP), IEEE International Conference on, 2002, pp. 1781-1784

[4] T. Rahim and D. E. N. Davies, "Effect of directional elements on the directional response of circular antenna arrays," in Microwaves, Optics and Antennas, IEE Proceedings H, 1982, pp. 18-22.

[5] J. Meyer, "Beamforming for a circular microphone array mounted on spherically shaped objects," The Journal of the Acoustical Society of America, vol. 109, no. 1, pp. 185-193, 2001.

[6] I. Balmages and B. Rafaely, "Open-sphere designs for spherical microphone arrays," IEEE Transactions on Audio, Speech and Language Processing, vol. 15, no. 2, pp. 727-732, 2007.

[7] A. Parthy, C. T. Jin, and A. van Schaik, "Acoustic holography with a concentric rigid and open spherical microphone array," in Acoustics, Speech and Signal Processing (ICASSP), IEEE International Conference on, 2009, pp. 2173-2176.

[8] C. T. Jin, N. Epain, and A. Parthy, "Design, optimization and evaluation of a dual-radius spherical microphone array," Audio, Speech, and Language Processing, IEEE/ACM Transactions on, vol. 22, no. 1, pp. 193-204, Jan 2014.

[9] B. Rafaely, "The spherical-shell microphone array," IEEE Transactions on Audio, Speech and Language Processing, vol. 16, no. 4, pp. 740-747, May 2008.

[10] T. D. Abhayapala and A. Gupta, "Alternatives to spherical microphone arrays: Hybrid geometries," in Acoustics, Speech and Signal Processing (ICASSP), IEEE International Conference on, Apr. 2009, pp. 81-84.

[11] A. Gupta and T. D. Abhayapala, "Double sided cone array for spherical harmonic analysis of wavefields," in Acoustics, Speech and Signal Processing (ICASSP), IEEE International Conference on, 2010, pp. 77 80.

[12] D. L. Alon and B. Rafaely, "Spindle-torus sampling for an efficientscanning spherical microphone array," Acta Acustica United With Acustica, vol. 98, no. 1, pp. 83-90, Jan. 2012.

[13] R. Mignot, G. Chardon, and L. Daudet, "Low frequency interpolation of room impulse responses using compressed sensing," IEEE/ACM Transactions on Audio, Speech and Language Processing (TASLP), vol. 22, no. 1, pp. 205-216, 2014.

[14] B. Rafaely, "Bessel nulls recovery in spherical microphone arrays for time-limited signals," Audio, Speech, and Language Processing, IEEE Transactions on, vol. 19, no. 8, pp. 2430-2438, 2011.

[15] G. Chardon, W. Kreuzer, and M. Noisternig, "Design of a robust open spherical microphone array," in Acoustics, Speech and Signal Processing (ICASSP), 2014 IEEE International Conference on, Florence, Italy, May 2014, pp. 6860-6863.

[16] H. L. Van Trees, Optimum Array Processing, ser. Part IV of Detection, Estimation, and Modulation Theory. Wiley Interscience, 2002.

[17] A. Moiola, R. Hiptmair, and I. Perugia, "Plane wave approximation of homogeneous Helmholtz solutions," Zeitschrift für Angewandte Mathematik und Physik (ZAMP), vol. 62, pp. 809-837, 2011.

[18] J. Melenk, "Operator adapted spectral element methods I: harmonic and generalized harmonic polynomials," Numerische Mathematik, vol. 84 pp. 35-69, 1999.

[19] C. Runge, "Über empirische Funktionen und die Interpolation zwischen äquidistanten Ordinaten," Zeitschrift für Mathematik und Physik, vol. 46, pp. 224-243, 1901.

[20] A. Cohen, M. A. Davenport, and D. Leviatan, "On the stability and accuracy of least squares approximations," Foundations of Computational Mathematics, vol. 13, no. 5, pp. 819-834, 2013
[21] G. Chardon, A. Cohen, and L. Daudet, "Reconstruction of solutions to the Helmholtz equation from punctual measurements," in 10th International Conference on Sampling Theory and Applications, Bremen, Germany, Jul. 2013

[22] —, "Sampling and reconstruction of solutions to the Helmholtz equation," Sampling Theory in Signal and Image Processing, vol. 13, no. 1 , pp. 67-89, 2014.

[23] I. H. Sloan and R. S. Womersley, "The Uniform Error of Hyperinterpolation on the Sphere," in Advances in Multivariate Approximation, W. Haußmann, K. Jetter, and M. Reimer, Eds. Witten-Bommerholz, Germany: Wiley-VCH Verlag, 1998, pp. 289-306.

[24] R. Womersley and I. Sloan, "How good can polynomial interpolation on the sphere be?" Advances In Computational Mathematics, vol. 14, no. 3, pp. 195-226, 2001

[25] Y. Maday, N. Nguyen, A. Patera, and G. Pau, "A general multipurpose interpolation procedure: The magic points," Comm. Pure Appl. Math., vol. 8 , no. 1, pp. 383-404, 2009.

[26] A. H. Barnett, "Dissipation in deforming chaotic billiards," Ph.D. dissertation, Harvard University, 2000.

[27] T. Betcke and L. Trefethen, "Reviving the method of particular solutions," SIAM review, pp. 469-491, 2005.

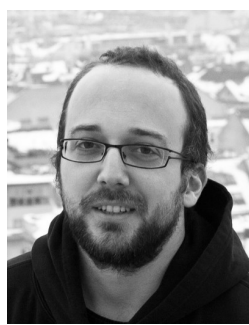

Gilles Chardon received the engineering degrees of the École Polytechnique and Telecom ParisTech in 2009, as well as the MSc ATIAM of Université Pierre et Marie Curie, Paris VI. After working towards his $\mathrm{PhD}$ at Institut Langevin in Paris, and a postdoctoral position with the Mathematics and Signal Processing group of the Acoustics Research Institute of the Austrian Academy of Sciences in Vienna, he is now Associate Professor with CentraleSupélec, Gif-sur-Yvette, France. His main research interests include sparse representation of acoustical fields, inverse problems and numerical analysis in acoustics.

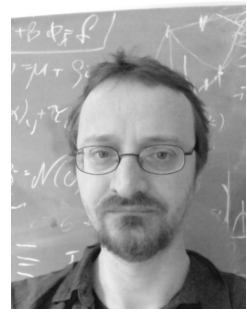

Wolfgang Kreuzer received his $\mathrm{Ph} . \mathrm{D}$ in engineering mathematics in 2000 at the Technical University of Vienna. After working at the Institute for Applied and Numerical Mathematics at the TU-Vienna he joined the Acoustics Research Institute of the Austrian Academy of Sciences in 2004. His main research interests is focused on models and simulation of wave propagation and wave scattering.

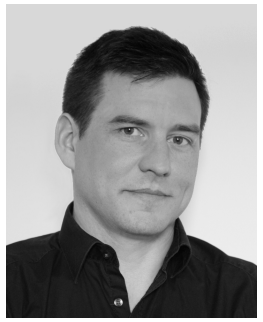

Markus Noisternig obtained his MSc degree in Electrical Engineering and Audio Engineering from the University of Technology (TUG) and the University of Music and Performing Arts (KUG) Graz in 2003. From 2003 to 2007 he was a Research Scientist at the Institute of Electronic Music and Acoustics (IEM) working towards his $\mathrm{PhD}$. In 2007 he was researcher at the LIMSI-CNRS and since 2008, he has been a Researcher at the UMR IRCAM-CNRSUPMC in Paris. He is also Senior Lecturer at the IEM and lectures at the Hochschule für Gestaltung in Karlsruhe. Taking both an artistic and scientific interest in matters of sound and music, he also studied computer music composition at the KUG. 